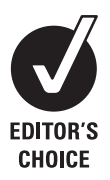

University of Sheffield, Sheffield, UK; ${ }^{2}$ Sheffield Teaching Hospitals Foundation Trust, Sheffield, UK; ${ }^{3}$ Northern Lincolnshire and Goole Foundation Trust, UK:

${ }^{4}$ University Hospital Aintree Hospitals NHS Trust, Liverpool, UK; ${ }^{5}$ Whiston Hospital, Merseyside, UK; ${ }^{6}$ Warrington Hospital, Warrington, UK;

${ }^{7}$ Taunton \& Somerset Hospital Foundation Trust, Taunton \& Somerset Hospital Foundation Trust, Taunton, UK:

${ }^{8}$ Worcestershire Royal Hospital, Worcester, UK; ${ }^{9}$ West Cumberland Hospital, Cumbria UK

\section{Correspondence to:}

Prof Steve Goodacre, Medical

Care Research Unit, Regent

Court, 30 Regent Street

Sheffield S1 4DA, UK;

s.goodacre@sheffield.ac.uk

Accepted 17 March 2009

\title{
Clinical diagnosis of acute coronary syndrome in patients with chest pain and a normal or non- diagnostic electrocardiogram
}

\author{
S Goodacre, ${ }^{1}$ P Pett, ${ }^{1}$ J Arnold, ${ }^{2}$ A Chawla, ${ }^{3}$ J Hollingsworth, ${ }^{4}$ D Roe, ${ }^{5}$ S Crowder, ${ }^{6}$ \\ C Mann, ${ }^{7}$ D Pitcher, ${ }^{8}$ C Brett $^{9}$
}

\begin{abstract}
Background: Clinical features may be used to determine which patients with suspected acute coronary syndrome (ACS), but a normal or non-diagnostic ECG, should be selected for further investigation or inpatient care. We aimed to measure the diagnostic value of clinical features for ACS
\end{abstract}

Methods: Standardised data relating to presenting characteristics, associated features and risk factors were collected at seven chest pain units established for the ESCAPE trial. All patients received troponin measurement at least $6 \mathrm{~h}$ after last significant symptoms, creatine kinase $\mathrm{MB}$ (mass) gradient over $2 \mathrm{~h}$ and, if appropriate, treadmill exercise testing. The reference standard of ACS was defined as troponin $>0.03 \mathrm{ng} / \mathrm{ml}$, creatine kinase $\mathrm{MB}$ (mass) gradient $>3.0 \mathrm{ng} / \mathrm{ml}$ or early positive treadmill exercise test.

Results: 1576 patients were analysed, including 132 $(8.4 \%)$ with ACS. Patients with ACS were older, had longer symptom duration, were more likely to be a man, hypertensive and an ex-smoker or have pain radiating to their right arm. On multivariate analysis, only age, duration, sex and radiation of pain to the right arm were independently associated with ACS. Likelihood ratios (95\% Cl) were radiation of pain to the right arm, 2.9 (95\% $\mathrm{Cl} 1.4$ to 6.3), male sex $1.2(95 \% \mathrm{Cl} 1.0$ to 1.3$)$ and female sex 0.79 (95\% Cl 0.62 to 1.0). The area under the receiver operator characteristic curve for age was 0.629 (95\% Cl 0.573 to 0.686$)$ and for duration was 0.546 (95\% Cl 0.481 to 0.610$)$.

Conclusion: Clinical features have very limited value for diagnosing ACS in patients with a normal or nondiagnostic ECG. Radiation of pain to the right arm increases the likelihood of ACS.

Acute chest pain is one of the most common diagnostic challenges in emergency medicine. ${ }^{1}$ Diagnosis principally focuses on identifying patients with acute coronary syndrome (ACS) who are at significant risk of adverse outcome and have the potential to benefit from inpatient care. The ECG provides a quick, cheap and simple way of identifying patients with ST segment changes who are likely to benefit from admission and is therefore an essential tool for evaluating any patient with suspected ACS. ${ }^{2}$ However, some patients with chest pain and a normal or nondiagnostic ECG may also be at significant risk of adverse outcome.

Biochemical cardiac markers, particularly troponins, can identify which patients with a normal or non-diagnostic ECG are at higher risk. ${ }^{3}$ For selected patients, treadmill exercise testing can provide further prognostic information. ${ }^{4}$ It is possible to provide at least biochemical cardiac marker testing for all patients with a normal or non-diagnostic ECG, but this is inconvenient for patients and potentially costly and may lead to emergency department overcrowding and/or substantial admissions to hospitals or observation units. It would therefore be helpful to identify any specific clinical features that could be useful in selecting patients for further investigation.

Numerous studies have evaluated clinical features in acute chest pain. Systematic reviews and meta-analysis have identified several clinical features that appear to be useful for establishing the probability of ACS. ${ }^{25}$ Table 1 reports likelihood ratios from these studies. The likelihood ratio estimates the diagnostic value of each piece of information by indicating how much more likely (or less likely) a diagnosis of ACS is as a result of the clinical finding. The higher the likelihood ratio above 1 , the more useful the finding is for ruling in ACS. The lower the likelihood ratio below 1, the more useful the finding is for ruling out ACS.

The primary studies for these reviews have two important limitations that should be taken into account before applying their findings to patients with a normal or non-diagnostic ECG. First, most of the studies included patients with ECG abnormalities. Clinical features that simply help to confirm the diagnosis of ACS in a patient with a diagnostic ECG are likely to have limited practical value. Second, most of the studies used the old World Health Organization (WHO) criteria for the diagnosis of acute myocardial infarction as the reference standard. The availability and use of troponin measurement have identified a substantial number of patients with ACS who are at significant risk of adverse outcome but do not have myocardial infarction according to the old WHO criteria. A few studies have selected patients with normal or non-diagnostic ECG and used broader criteria for ACS than the WHO criteria for myocardial infarction. ${ }^{6}$ These have shown that clinical features appear to have limited diagnostic value in the patient group in which they are most likely to be used to guide decision making.

The ESCAPE trial (Effectiveness and Safety of Chest pain Assessment to Prevent Emergency admissions) involved setting up chest pain units (CPUs) at seven diverse hospitals to manage patients with acute chest pain but a normal or non-diagnostic ECG. ${ }^{8}$ As part of the evaluation, 
Table 1 Likelihood ratios for clinical features used to diagnose ACS

\begin{tabular}{ll}
\hline Clinical feature & Likelihood ratio \\
\hline Radiation of pain to the right arm or shoulder & 4.7 \\
Radiation of pain to both arms or shoulders & 4.1 \\
Associated with exertion & 2.4 \\
Radiation of pain to the left arm & 2.3 \\
Associated with diaphoresis & 2.0 \\
Associated with nausea or vomiting & 1.9 \\
Worse than angina/similar to previous myocardial infarction & 1.8 \\
Described as pressure & 1.3 \\
Described as pleuritic & 0.2 \\
Described as positional & 0.3 \\
Described as sharp & 0.3 \\
Reproduced by palpation & 0.3 \\
Not associated with exertion & 0.8 \\
\hline
\end{tabular}

standardised data were collected on all patients receiving CPU care. We therefore planned to use these data to evaluate the diagnostic value of clinical features for ACS in patients with chest pain and a normal or non-diagnostic ECG.

\section{METHODS}

The ESCAPE trial was a cluster-randomised trial in which 14 hospitals were randomly allocated to either establish CPU care or continue with routine care. ${ }^{8}$ The seven hospitals randomised to intervention all successfully established CPU care and collected data from patients managed by the CPU over 1 year. The structures, processes and the outcomes of the CPUs have been described previously. ${ }^{9}$ Essentially, they were all based on a common protocol in which low-risk patients with acute chest pain were investigated by a combination of creatine kinase $\mathrm{MB}$ (mass) (CK-MB(mass)) and troponin testing, followed by treadmill exercise testing for selected patients. Those with positive findings for ACS were admitted to hospital, whereas those with negative tests were discharged home.

Patients attending the emergency department with acute chest pain of possible cardiac ischaemic origin were eligible for CPU care unless they met the following exclusion criteria: (1) ECG changes diagnostic for ACS ( $>1 \mathrm{~mm}$ ST segment deviation or $>3 \mathrm{~mm} \mathrm{~T}$ wave inversion), (2) known coronary heart disease with recurrent or prolonged episodes of their typical anginal pain, (3) suspected or proven life-threatening non-cardiac pathology (eg, pulmonary embolus or aortic dissection and (4) comorbidities requiring inpatient care (eg, arrhythmia or heart failure).

The CPU protocol consisted of CK-MB(mass) measurement at baseline and at least $2 \mathrm{~h}$ and troponin measurement at least $6 \mathrm{~h}$ after the last significant episode of pain. Patients presenting $12 \mathrm{~h}$ after their worst pain were tested with a single sample for troponin. Following this, patients underwent treadmill exercise testing, either immediately or on the next working day, unless they were unable to perform a treadmill test or had recently had provocative cardiac testing or cardiac imaging. Previous studies have shown that these tests identify ACS reliably in isolation and in combination. ${ }^{10-15}$

Doctors or specialist chest pain nurses collected data on a standardised form from all patients managed according to the CPU protocol. The data collection form recorded presenting clinical characteristics, risk factors for coronary heart disease and the results of CPU diagnostic testing. A clinical researcher (JA) working with the ESCAPE trial reviewed all data collection forms, checked data accuracy and obtained missing data, where possible, by reference to the inpatient notes. The primary purpose of data collection was to audit each CPU. Anonymised data from the form were entered on to an Excel spreadsheet by JA, and summaries of these data were reported to the Trial Data Monitoring Committee.

We used the anonymised data to undertake this secondary analysis. Cases were excluded if they did not have a troponin measurement during their CPU assessment. This occurred most commonly because they were admitted to the hospital with further symptoms or complications, although some patients self-discharged before completing assessment. We used the following criteria to define the reference standard diagnosis of ACS: (1) troponin concentration $>0.03 \mathrm{ng} / \mathrm{ml}$, (2) CK$\mathrm{MB}$ (mass) gradient of $>3.0 \mathrm{ng} / \mathrm{ml}$ or (3) early positive treadmill exercise test. The treadmill test was performed by either cardiac technicians or chest pain nurses and interpreted by a single doctor interpreter who was not blind to patients' presenting characteristics. The test was considered positive if there was $>1 \mathrm{~mm}$ ST elevation or depression in any two contiguous leads. Clinical characteristics were recorded prior to diagnostic testing and thus blind to the criteria for ACS diagnosis.

Data were analysed using SPSS for Windows V.15. Logistic regression was used to test univariate associations between each categorical variable and ACS. $t$ Tests were used to test univariate associations between each continuous variable and ACS. We then entered each variable that showed a univariate association with ACS $(p<0.1)$ into a multivariate logistic regression model. The diagnostic value of each variable was then assessed by calculating likelihood ratios for categorical variables, using Confidence Interval Analysis software and constructing a receiver operator characteristic curve for continuous variables, using SPSS for Windows V.15.

\section{RESULTS}

The ESCAPE intervention hospitals collected data from 1644 patients. We excluded 68 cases that did not receive a troponin measurement during their CPU assessment and therefore analysed data from 1576 patients (56.8\% men, mean age 53 years (SD 14, range 17-98 years)). Overall, 132 (8.4\%) of 1576 were diagnosed as having ACS: 18 had both elevated troponin and positive CK- $\mathrm{MB}$ (mass) gradient, 32 had elevated troponin but negative $\mathrm{CK}-\mathrm{MB}$ (mass) gradient, two had a positive $\mathrm{CK}-\mathrm{MB}$ gradient but no troponin elevation and 27 patients had elevated troponin but insufficient CK-MB(mass) measurements to calculate a gradient. Thus, 79 of 1576 patients were diagnosed as having ACS on the basis of biochemical markers $(5.0 \%)$.

Treadmill exercise tests were performed on 1034/1576 (65.6\%). The reasons for not performing a treadmill test were: inability to perform (108), contraindication to treadmill testing (56), recent provocative testing or cardiac imaging (70), other reasons (122) and no reason recorded (186). Among those tested, $55 / 1034(5.3 \%)$ had an early positive treadmill exercise test. Two of these had undergone treadmill testing despite having a small troponin elevation recorded. Thus a total of 132/1576 patients were diagnosed as having ACS (8.4\%).

The proportion of the cohort with each categorical feature and the association between each categorical feature and ACS are shown in table 2. The associations between continuous variables (age and pain duration) and ACS are shown in table 3. Patients with ACS tended to be older and have a longer duration of pain; were more likely to be a man, hypertensive or an ex-smoker and more likely to have pain 
Table 2 Univariate analysis of the association between categorical variables and ACS

\begin{tabular}{|c|c|c|c|}
\hline Feature & $\begin{array}{l}\text { With the feature } \\
(\mathrm{n} / \mathrm{N}(\%))\end{array}$ & $\begin{array}{l}\text { OR for ACS } \\
(95 \% \mathrm{CI})\end{array}$ & p Value \\
\hline Male sex & $895 / 1576(56.7 \%)$ & 1.47 (1.01 to 2.13$)$ & 0.044 \\
\hline \multicolumn{4}{|l|}{ Nature of chest pain } \\
\hline Indigestion/burning & $135 / 1555(8.7 \%)$ & 1.89 (0.85 to 4.23$)$ & 0.119 \\
\hline Stabbing/sharp & $311 / 1555$ (20.0\%) & 0.87 (0.40 to 1.88$)$ & 0.715 \\
\hline Aching/dull/heavy & $711 / 1555$ (45.7\%) & 1.27 (0.65 to 2.48$)$ & 0.475 \\
\hline Gripping/crushing & $232 / 1555$ (14.9\%) & $1.70(0.81$ to 3.56$)$ & 0.159 \\
\hline Non-specific/other & $166 / 1555$ (10.7\%) & Reference & - \\
\hline \multicolumn{4}{|l|}{ Site of chest pain } \\
\hline Central & 1108/1564 (70.8\%) & $0.90(0.47$ to 1.74$)$ & 0.760 \\
\hline Left chest & $308 / 1564$ (19.7\%) & $0.56(0.25$ to 1.22$)$ & 0.145 \\
\hline Right chest & $36 / 1564$ (2.3\%) & 0.26 (0.03 to 2.06$)$ & 0.201 \\
\hline Other & 110/1564 (7.0\%) & Reference & - \\
\hline \multicolumn{4}{|l|}{ Radiation of chest pain } \\
\hline None & $605 / 1534(39.2 \%)$ & Reference & - \\
\hline Left arm & 456/1534 (29.7\%) & 1.43 (0.92 to 2.21$)$ & 0.108 \\
\hline Right arm & $39 / 1534(2.5 \%)$ & 3.92 (1.75 to 8.79$)$ & 0.001 \\
\hline Neck & 78/1534 (5.1\%) & 0.89 (0.34 to 2.33$)$ & 0.821 \\
\hline Jaw & $60 / 1534$ (3.9\%) & $0.69(0.21$ to 2.29$)$ & 0.542 \\
\hline Back & 174/1534 (11.3\%) & 0.88 (0.44 to 1.75$)$ & 0.719 \\
\hline Other & $122 / 1534(8.0 \%)$ & $1.69(0.90$ to 3.20$)$ & 0.105 \\
\hline \multicolumn{4}{|l|}{ Time of onset } \\
\hline 00:00-05:59 & $254 / 1409$ (18.0\%) & 1.06 (0.58 to 1.94$)$ & 0.853 \\
\hline 06:00-11:59 & 675/1409 (47.9\%) & $0.74(0.43$ to 1.27$)$ & 0.276 \\
\hline $12: 00-17: 59$ & $264 / 1409$ (18.7\%) & $0.93(0.50$ to 1.72$)$ & 0.813 \\
\hline 18:00-23:59 & $216 / 1409$ (15.3\%) & Reference & - \\
\hline Continuous & $873 / 1303(67.0 \%)$ & 0.98 (0.65 to 1.49$)$ & 0.938 \\
\hline Nausea & 489/1521 (32.1\%) & $1.09(0.74$ to 1.60$)$ & 0.667 \\
\hline Vomiting & $92 / 1501(6.1 \%)$ & 1.21 (0.59 to 2.47$)$ & 0.603 \\
\hline Sweating & $644 / 1521$ (42.3\%) & $1.18(0.82$ to 1.70$)$ & 0.370 \\
\hline Dyspnoea & $608 / 1507(40.3 \%)$ & 0.94 (0.64 to 1.36$)$ & 0.728 \\
\hline Previous coronary heart disease & 196/1534 (12.8\%) & $0.98(0.57$ to 1.70$)$ & 0.950 \\
\hline Diabetes & $94 / 1524(6.2 \%)$ & 0.87 (0.39 to 1.92$)$ & 0.731 \\
\hline Hypertension & 459/1511 (30.4\%) & 1.62 (1.12 to 2.34$)$ & 0.011 \\
\hline Hyperlipidaemia & 400/1418 (28.2\%) & 1.17 (0.78 to 1.77$)$ & 0.428 \\
\hline Smoker & $478 / 1512(31.6 \%)$ & 0.85 (0.57 to 1.28$)$ & 0.443 \\
\hline Ex-smoker & $207 / 1449(14.3 \%)$ & $1.70(1.07$ to 2.69$)$ & 0.025 \\
\hline Cocaine use & $23 / 1445(0.1 \%)$ & Not calculated* & 0.998 \\
\hline Family history & 645/1422 (45.4\%) & $0.80(0.55$ to 1.17$)$ & 0.251 \\
\hline Chest wall & $82 / 1367(6.0 \%)$ & 0.58 (0.21 to 5.62$)$ & 0.301 \\
\hline
\end{tabular}

ACS, acute coronary syndrome; $\mathrm{OR}$, odds ratio.

radiating to their right arm. All other features were not significantly associated with ACS.

The six variables with univariate association with ACS were entered into a multivariate model. The results are shown in table 4. Older age, male sex, longer duration of pain and radiation of pain to the right arm were independent predictors of ACS. Hypertension and previous smoking were not associated with ACS on multivariate analysis.

The area under the receiver operator characteristic curve for age was 0.629 (95\% CI 0.573 to 0.686 ) and for duration was
$0.546(95 \%$ CI 0.481 to 0.610$)$. Sensitivity, specificity and likelihood ratios for all variables are reported in table 5 .

\section{DISCUSSION}

This study reports one of the largest cohorts evaluating the diagnostic value of clinical features for ACS. The findings show that in patients with a normal or non-diagnostic ECG, no serious comorbidity and no obvious alternative cause for their symptoms, there are few diagnostically useful clinical features.

Table 3 Univariate analysis of continuous variables

\begin{tabular}{lllr}
\hline Feature & With ACS & Without ACS & p Value \\
\hline Age (years) & $58.8(13.2)$ & $52.2(13.9)$ & $<0.001$ \\
Duration of pain (minutes) & $53.6(53.3)$ & $43.2(43.6)$ & 0.030 \\
\hline ACS, acute coronary syndrome. & & \\
Values are mean (SD). & &
\end{tabular}


Table 4 Results of multivariate analysis

\begin{tabular}{llr}
\hline Feature & OR (95\% CI) & p Value \\
\hline Age & $1.034(1.017$ to 1.051$)$ & $<0.001$ \\
Sex & $1.71(1.07$ to 2.74$)$ & 0.024 \\
Duration of chest pain & $1.005(1.000$ to 1.009$)$ & 0.040 \\
Radiation of pain to the & $5.82(2.38$ to 14.2$)$ & $<0.001$ \\
right arm & & \\
Hypertension & $1.23(0.77$ to 1.96$)$ & 0.385 \\
Ex-smoker & $1.35(0.75$ to 2.42$)$ & 0.310
\end{tabular}

Age, sex, duration of symptoms and radiation of pain to the right arm were the only independent predictors of ACS. Of these, arguably only radiation of pain to the right arm, with a likelihood ratio of 2.9, provides diagnostic information that might influence clinical decision making.

Systematic reviews of previous studies have identified a number of diagnostically useful clinical features, as outlined in table $1 .{ }^{25}$ This study confirms that radiation of pain to the right arm appears to be the most powerful indicator of ACS. This contrasts with traditional teaching that radiation of pain to the left arm is typical of cardiac pain. One possible explanation is that patients are aware that radiation of pain to the left arm suggests cardiac pain and so attend the emergency department with any pain radiating to the left arm but attend with pain radiating to the right arm only if it is exceptionally severe or unexplained by other factors.

Other features previously shown to be diagnostically useful were not supported by our study. Nausea, vomiting, sweating, radiation of pain to the left $\mathrm{arm}^{5}$ and burning-type ${ }^{7}$ pain have previously been shown to predict ACS, while stabbing pain and pain reproduced by palpation have been shown to reduce the likelihood of ACS. ${ }^{5}$ The odds ratios for these features in our analysis showed weak trends in the same direction as those in previous studies, but these were not significant on univariate analysis. This suggests that our study may not have had sufficient power to detect an association for these variables, and/or the associations between these variables and ACS are weaker in a cohort of patients with normal or non-diagnostic ECG.

Table 5 Sensitivity, specificity and likelihood ratios for each variable

\begin{tabular}{|c|c|c|c|c|}
\hline Feature & Sensitivity & Specificity & $\begin{array}{l}\text { Positive likelihood } \\
\text { ratio }\end{array}$ & $\begin{array}{l}\text { Negative likelihood } \\
\text { ratio }\end{array}$ \\
\hline Male sex & $86 / 132(65.2 \%)$ & $635 / 1444$ (44.0\%) & 1.16 (1.02 to 1.33$)$ & $0.792(0.623$ to 1.01$)$ \\
\hline \multicolumn{5}{|l|}{ Nature of chest pain } \\
\hline Indigestion/burning & $16 / 129(12.4 \%)$ & $1307 / 1426(91.7 \%)$ & $1.49(0.91$ to 2.43$)$ & $0.956(0.894$ to 1.02$)$ \\
\hline Stabbing/sharp & $18 / 129(14.0 \%)$ & $1133 / 1426(79.5 \%)$ & $0.679(0.437$ to 1.06$)$ & $1.08(1.0$ to 1.17$)$ \\
\hline Aching/dull/heavy & $59 / 129(45.7 \%)$ & $774 / 1426$ (54.3\%) & $1.0(0.822$ to 1.22$)$ & $1.0(0.847$ to 1.18$)$ \\
\hline Gripping/crushing & $25 / 129(19.4 \%)$ & $1219 / 1426(85.5 \%)$ & $1.33(0.919$ to 1.94$)$ & $0.943(0.864$ to 1.03$)$ \\
\hline Non-specific/other & $11 / 129(8.5 \%)$ & $1271 / 1426(89.1 \%)$ & $0.784(0.437$ to 1.41$)$ & $1.03(0.971$ to 1.08$)$ \\
\hline \multicolumn{5}{|l|}{ Site of chest pain } \\
\hline Central & $101 / 131$ (77.7\%) & $424 / 1431$ (29.6\%) & $1.10(0.992$ to 1.21$)$ & $0.773(0.559$ to 1.07$)$ \\
\hline Left chest & $18 / 131(13.7 \%)$ & $1141 / 1431$ (79.7\%) & $0.678(0.436$ to 1.05$)$ & $1.08(1.01$ to 1.16$)$ \\
\hline Right chest & $1 / 131(0.8 \%)$ & $1396 / 1431$ (97.6\%) & $0.312(0.043$ to 2.26$)$ & $1.02(1.0$ to 1.04$)$ \\
\hline Other & $11 / 131$ (8.4\%) & $1332 / 1431(93.1 \%)$ & 1.21 (0.668 to 2.20$)$ & $0.984(0.933$ to 1.04$)$ \\
\hline \multicolumn{5}{|l|}{ Radiation of chest pain } \\
\hline None & $43 / 130(33.1 \%)$ & $842 / 1404$ (60.0\%) & $0.826(0.642$ to 1.06$)$ & $1.12(0.982$ to 1.27$)$ \\
\hline Left arm & $45 / 130(34.6 \%)$ & 993/1404 (70.7\%) & $1.18(0.921$ to 1.52$)$ & $0.924(0.812$ to 1.05$)$ \\
\hline Right arm & $9 / 130(6.9 \%)$ & $1374 / 1404$ (97.9\%) & 3.24 (1.57 to 6.68$)$ & 0.951 (0.907 to 0.997$)$ \\
\hline Neck & $5 / 130(3.8 \%)$ & $1331 / 1404$ (94.8\%) & $0.740(0.304$ to 1.80$)$ & $1.01(0.978$ to 1.05$)$ \\
\hline Jaw & $3 / 130(2.3 \%)$ & $1347 / 1404(95.9 \%)$ & $0.568(0.181$ to 1.79$)$ & $1.02(0.990$ to 1.05$)$ \\
\hline Back & $11 / 130(8.5 \%)$ & $1241 / 1404(88.4 \%)$ & $0.729(0.407$ to 1.31$)$ & $1.04(0.980$ to 1.09$)$ \\
\hline Other & $14 / 130(10.8 \%)$ & $1296 / 1404(92.3 \%)$ & $1.40(0.827$ to 2.37$)$ & $0.967(0.909$ to 1.03$)$ \\
\hline \multicolumn{5}{|l|}{ Time of onset } \\
\hline $00: 00-05: 59$ & $26 / 121(21.5 \%)$ & $1060 / 1288(82.3 \%)$ & $1.21(0.847$ to 1.74$)$ & $0.954(0.866$ to 1.05$)$ \\
\hline $06: 00-11: 59$ & $50 / 121(41.3 \%)$ & $663 / 1288(51.5 \%)$ & $0.852(0.684$ to 1.06$)$ & $1.14(0.973$ to 1.34$)$ \\
\hline $12: 00-17: 59$ & 24/121 (19.8\%) & $1048 / 1288(81.4 \%)$ & $1.06(0.731$ to 1.55$)$ & $0.985(0.898$ to 1.08$)$ \\
\hline $18: 00-23: 59$ & $21 / 121(17.4 \%)$ & $1129 / 1288(87.7 \%)$ & $1.41(0.928$ to 2.13$)$ & $0.943(0.867$ to 1.03$)$ \\
\hline Continuous & $74 / 111(66.7 \%)$ & $393 / 1192$ (33.0\%) & $0.995(0.867$ to 1.14$)$ & $1.01(0.768$ to 1.33$)$ \\
\hline Nausea & $43 / 127(33.9 \%)$ & $948 / 1394$ (68.0\%) & $1.06(0.820$ to 1.36$)$ & $0.973(0.854$ to 1.11$)$ \\
\hline Vomiting & $9 / 125(7.2 \%)$ & $1293 / 1376(94.0 \%)$ & $1.19(0.615$ to 2.32$)$ & $0.988(0.939$ to 1.04$)$ \\
\hline Sweating & $59 / 128(46.1 \%)$ & $808 / 1393$ (58.0\%) & $1.10(0.909$ to 1.34$)$ & $0.929(0.787$ to 1.10$)$ \\
\hline Dyspnoea & $49 / 126(38.9 \%)$ & $822 / 1381$ (59.5\%) & $0.961(0.765$ to 1.21$)$ & $1.03(0.887$ to 1.19$)$ \\
\hline Previous coronary heart disease & $16 / 127(12.6 \%)$ & $1227 / 1407$ (87.2\%) & 0.985 (0.611 to 1.59$)$ & $1.0(0.935$ to 1.07$)$ \\
\hline Diabetes & $7 / 128(5.5 \%)$ & $1309 / 1396(93.8 \%)$ & $0.878(0.415$ to 1.85$)$ & $1.01(0.965$ to 1.05$)$ \\
\hline Hypertension & $52 / 129(40.3 \%)$ & $975 / 1382(70.5 \%)$ & 1.37 (1.09 to 1.71$)$ & $0.846(0.731$ to 0.979$)$ \\
\hline Hyperlipidaemia & $37 / 118(31.4 \%)$ & $937 / 1300(72.1 \%)$ & 1.12 (0.848 to 1.49$)$ & $0.952(0.839$ to 1.08$)$ \\
\hline Smoker & $36 / 126(28.6 \%)$ & $944 / 1386$ (68.1\%) & $0.896(0.673$ to 1.19$)$ & $1.05(0.934$ to 1.79$)$ \\
\hline Ex-smoker & $26 / 123(21.1 \%)$ & $1145 / 1326(86.3 \%)$ & 1.55 (1.07 to 2.24$)$ & $0.913(0.831$ to 1.0$)$ \\
\hline Cocaine use & $0 / 122(0 \%)$ & $1300 / 1323$ (98.3\%) & - & - \\
\hline Family history & $48 / 119(40.3 \%)$ & 706/1303 (54.2\%) & $0.880(0.702$ to 1.10$)$ & 1.10 (0.942 to 1.29$)$ \\
\hline Chest wall & 4/108 (3.7\%) & $1181 / 1259(93.8 \%)$ & $0.598(0.223$ to 1.60$)$ & $1.03(0.987$ to 1.07$)$ \\
\hline
\end{tabular}

Differences in denominators for sensitivity and specificity are due to missing data. 
This study has a number of limitations that should be taken into account when interpreting the findings. These limitations principally relate to the fact that this study was a secondary analysis of data collected for another purpose. There was no sample size calculation, and the study was not powered to detect potentially important associations between clinical features and ACS. Data collection had to be feasible for the clinical staff responsible for undertaking this role, so we did not examine all potentially useful clinical features comprehensively. For the same reason, we were unable to examine interobserver error. Furthermore, although the reference standard was pragmatic and reasonably robust, it is possible that some cases with and without ACS may have been misclassified. In particular, the choice of threshold for CK-MB(mass) gradient was somewhat arbitrary, reflecting a compromise between the value of this measure in early detection of ACS and the risk of a false-positive CK- $\mathrm{MB}$ (mass) increase, the use of a 6-hour protocol could have missed some patients with a small, late troponin increase, and patients with a positive treadmill test may have had significant coronary artery disease but not necessarily ACS.

Finally, it should be recognised that although the study population was reasonably well defined and relevant to clinical practice, it was selected from the wider population with chest pain on the basis of diagnostic uncertainty. Patients with clearly non-cardiac chest pain, such as musculoskeletal chest pain or anxiety-related chest pain, may not have been included in the study cohort, so our findings may not apply to patients with a clear non-cardiac diagnosis. Conversely, our findings should not be generalised to higher risk patients, such as those with known coronary heart disease.

The implication of this study is that the individual clinical features that we examined have very limited value in diagnosing ACS in patients with a normal or non-diagnostic ECG and cannot be used reliably to select patients for diagnostic testing or hospital admission. The decision regarding whether to use diagnostic testing or hospital admission for these patients depends on weighing the costs, risks and benefits of the various alternatives. If testing or admission is considered worthwhile, then it should be applied to all patients with suspected ACS.
Acknowledgements: We thank all the staff at Scunthorpe General Hospital, University Hospital Aintree, Whiston Hospital, Taunton and Somerset Hospital, West Cumberland Hospital, Warrington Hospital and Worcestershire Royal Hospital who were involved in the ESCAPE trial.

Funding: The ESCAPE trial was funded by the NHS Service Delivery and Organisation RGD Programme.

Competing interests: None.

Provenance and peer review: Not commissioned; externally peer reviewed.

\section{REFERENCES}

1. Goodacre $\mathbf{S}$, Cross E, Arnold J, et al. The health care burden of acute chest pain. Heart 2005;91:229-30.

2. Panju AA, Hemmelgarn BR, Guyatt $\mathrm{GH}$, et al. The rational clinical examination. Is this patient having a myocardial infarction? JAMA 1998;280:1256-63.

3. Antman E, Tanasijevic MJ, Thompson B, et al. Cardiac-specific troponin I levels to predict the risk of mortality in patients with acute coronary symptoms. N Engl J Med 1996;335:1342-9. Grade A.

4. Kirk JD, Turnipseed S, Lewis RL, et al. Evaluation of chest pain in low-risk patients presenting to the emergency department: the role of immediate exercise testing. Ann Emerg Med 1998;32:1-7.

5. Swap CT, Nagurney JT. Value and limitations of chest pain history in the evaluation of patients with suspected acute coronary syndromes. JAMA 2005;294:2623-9.

6. Goodacre S, Locker T, Morris F, et al. How useful are clinical features in the diagnosis of acute, undifferentiated chest pain? Acad Emerg Med 2002;9:203-8.

7. Goodacre SW, Angelini K, Arnold J, et al. Clinical predictors of acute coronary syndrome in patients with undifferentiated chest pain. O J Med 2003;96:893-8.

8. Goodacre S, Cross E, Lewis C, Nicholl J, Capewell S, on behalf of the ESCAPE Research Team. The ESCAPE cluster randomised trial: Effectiveness and Safety of Chest pain Assessment to Prevent Emergency admissions. BMJ 2007;335:659-62

9. Arnold J, Goodacre S, Morris F, on behalf of the ESCAPE Research Team. The structure, processes and outcomes of chest pain units established in the ESCAPE trial. Emerg Med J 2007;24:462-6.

10. Herren KR, Mackway-Jones K, Richards CR, et al. Is it possible to exclude a diagnosis of myocardial damage within six hours of admission to an emergency department? Diagnostic cohort study. BMJ 2001:323:372-4.

11. Fesmire FM, Percy RF, Bardoner JB, et al. Serial creatinine kinase (CK) MB testing during the emergency department evaluation of chest pain: utility of a 2-hour deltaCK-MB of $+1.6 \mathrm{ng} / \mathrm{ml}$. Am Heart J 1998;136:237-44.

12. Fesmire FM, Christensen RH, Focy EP, et al. Delta creatinine kinase MB outperforms myoglobin at two hours during the emergency department identification and exclusion of troponin positive non-ST elevation acute coronary syndromes. Ann Emerg Med 2004;44:12-9.

13. Hamm CW, Goldman BW, Heeschen C, et al. Emergency room triage of patients with acute chest pain by means of rapid testing for cardiac troponin T or troponin I. N Engl J Med 1997;337:1648-53.

14. Goodacre S, Nicholl J, Dixon S, et al. Randomised controlled trial and economic evaluation of a chest pain observation unit compared with routine care. BMJ 2004;328:254-7.

15. Collinson PO, Gaze DC, Morris F, et al. Comparison of biomarker strategies for rapid rule out of myocardial infarction in the emergency department using ACC/ESC diagnostic criteria. Ann Clin Biochem 2006;43:273-80. 